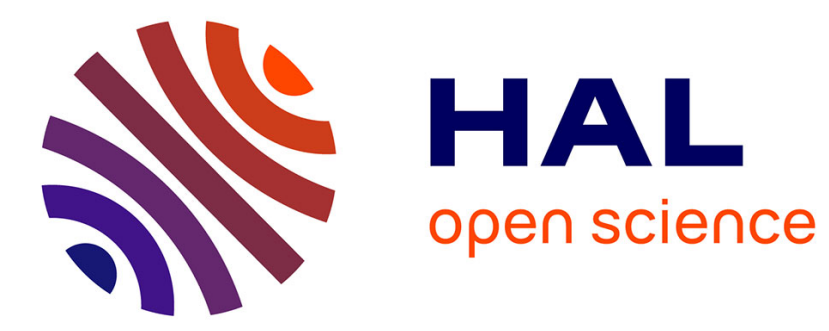

\title{
Dictionary learning based sinogram inpainting for CT sparse reconstruction
}

Si Li, Yang Chen, Limin Luo, Christine Toumoulin

\section{To cite this version:}

Si Li, Yang Chen, Limin Luo, Christine Toumoulin. Dictionary learning based sinogram inpainting for CT sparse reconstruction. optik- International Journal for Light and Electron Optics, 2014, 125 (12), pp.2862-2867. 10.1016/j.jjleo.2014.01.003 . inserm-00955275

\section{HAL Id: inserm-00955275 https://www.hal.inserm.fr/inserm-00955275}

Submitted on 4 Mar 2014

HAL is a multi-disciplinary open access archive for the deposit and dissemination of scientific research documents, whether they are published or not. The documents may come from teaching and research institutions in France or abroad, or from public or private research centers.
L'archive ouverte pluridisciplinaire HAL, est destinée au dépôt et à la diffusion de documents scientifiques de niveau recherche, publiés ou non, émanant des établissements d'enseignement et de recherche français ou étrangers, des laboratoires publics ou privés. 


\title{
Dictionary Learning Based Sinogram Inpainting for CT Sparse Reconstruction
}

\author{
$\mathrm{Si} \mathrm{Li}^{1,2,3}$, Yang Chen ${ }^{1,2,3,4}$, Limin Luo ${ }^{1,2}$, Christine Toumoulin ${ }^{2,3,4}$ \\ 1. Laboratory of Image Science and Technology, Southeast University, Nanjing, China; \\ 2. Centre de Recherche en Information Biomedicale Sino-Francais (LIA CRIBs), Rennes, France; \\ 3. Laboratoire Traitement du Signal \& de l'Image (LTSI)-Université de Rennes 1, F-35042 Rennes, France; \\ 4. INSERM, UMR1099, F-35042, Rennes, France; \\ \{lisi20050401, chenyang.list, luo.list\}@seu.edu.cn \\ Christine.toumoulin@univ-rennes1.fr
}

\begin{abstract}
In CT (computed tomography), reconstruction from undersampling projection data is often ill-posed and suffers from severe artifact in the reconstructed images. To overcome this problem, this paper proposes a sinogram inpainting method based on recently rising sparse representation technology. In this approach, a dictionary learning based inpainting is used to estimate the missing projection data. The final image can be reconstructed by the analytic filtered back projection (FBP) reconstruction. We conduct experiments using both simulated and real phantom data. Compared to the comparative interpolation method, visual and numerical results validate the clinical potential of the proposed method.
\end{abstract}

Keywords: computed tomography (CT), under-sampling, dictionary learning, inpainting.

\section{Introduction}

How to reduce the radiation exposure of patients has always been an important concern since the introduction of computed tomography (CT) [1-3]. Image reconstruction from sparse sampled sinogram data enables rapid scanning with a reduced x-ray dose delivered to patients. However, because of measurement missing in the sparse sampling, the projection data is not sufficient for exact reconstruction, the application of standard analytic algorithms such as filtered back-projection (FBP) will lead to conspicuous artifact in the reconstructed images[4].

A widely used reconstruction algorithm for sparse sampling is the iterative total variation (TV) minimization algorithm which relies on the assumption that the main information of the object being imaged can be well represented by sparse gradients [5]. Some Markov random fields (MRF) based bayesian approaches were also applied to improve the iterative reconstructions by incorporating image information as priors [6-7]. The main drawback of these iterative methods is the high computation cost involved. Another solution is completing the sinogram data by inpainting the missing measurements [89], and then perform analytic FBP reconstruction using the completed sinogram data. In recent years there has been a growing interest in the study of sparse representation based dictionary learning (DL) in signal processing [10-18]. Some successful applications in medical imaging have been explored. In [19-20], DL based regularizations were incorporated into the iterative algorithms to improve MRI and CT reconstructions under sparse sampling. Also an modified DL algorithm was developed in [21] to denoise CT images with simulated Poisson noise. In [22], J.Shtok utilized dictionary learning based method to denoise the sinogram in low-dose CT.

In this paper, we apply DL method to inpaint the missing sinogram data caused by sparse scanning in CT. The dictionary is learned via the overlapping sinogram patches from a simulated phantom image, then the missing sinogram data is estimated by performing the DL based inpainting. The final image can be reconstructed by performing FBP algorithm using the inpainted sinogram. The proposed method is explained in detail in Section 2. In Section 3, we demonstrate the performance of our algorithm on simulated and real data. A conclusion and future work are given in Section 4. 


\section{$2 \quad$ Method}

Based on [23], the classical FBP algorithm can be formulated as follows:

$$
I\left(c_{x}, c_{y}\right)=\int_{0}^{\pi} p\left(c_{r}, \theta\right) * h\left(c_{r}\right)_{c_{r}=r \cos (\theta-\phi)} d \theta \approx \sum_{m=0}^{N_{\phi}} \Delta d \sum_{n=-N}^{N} p(n d, m \Delta) h\left(c_{r}-n d\right)
$$

where, $I$ is the reconstructed image with pixel index denoted by $\left(c_{x}, c_{y}\right), p$ is the projection data, $h$ is the transfer function, $\theta$ is the projection view, $c_{r}$ is the rotational coordinates, $r$ is the distance from the reconstructed point to the coordinate origin, $\phi$ is the angle from the positive direction of $\mathrm{x}$-axis to the vector which connect the origin to the reconstructed point , $d$ is the sampling interval, $N_{\phi}$ is the number of projection views, $\Delta$ is the angle increment, $N$ is the number of projection bins.

For a sparse signal $y \in R^{n}, y$ can be represented in a linear system $A x=y\left(A \in R^{n \times m}, x \in R^{m}\right.$, $y \in R^{n}, m$ and $n$ represent the dimension of the vector $x$ and $y$, respectively) [14]. The matrix $A$ refers to a dictionary of atoms, and every column in $A$ is a atomic signal in $R^{n} . x$ is a sparse vector with $\|x\|_{0}^{0}=k_{0}$, and \|\|$_{0}^{0}$ is the $l_{0}$ norm which represents the number of non-zeros in the vector. The product of $A$ by the sparse vector $x$ produces a linear combination of $k_{0}$ atoms with varying portions as the signal $y$, the vector $x$ that generates $y$ is called the sparse representation of $y$.

For our case, we assume that the original sinogram image $u \in R^{n}$ is a sparse-land signal, which means that there exists a dictionary $A$, and a corresponding sparse representation vector $\alpha$ allowing $u=A \alpha[10]$. The sparse measurement $Z$ can be defined as follows:

$$
Z=M u+\varepsilon
$$

where, $M$ denotes a binary mask matrix specifying the locations of the known or missing pixels, and $\varepsilon$ represents the noise. To estimate the original image $u$, we use the coefficient sparsity as a prior constraint, which can be formulated as:

$$
\min _{\alpha}\|\alpha\|_{0}^{0} \text { s.t. }\|Z-M A \alpha\|_{2}^{2} \leq \delta
$$

$\delta$ is a parameter that should be set proportional to the amount of noise, \|\|$_{0}^{0}$ and \|\|$_{2}^{2}$ represent the $l_{0}$ norm and $l_{2}$ norm, respectively. We can obtain the representation $\alpha$ by solving the problem (3), and then calculate the inpainted sinogram $u$ as $A \alpha$. Then with inpainted sinogram available, the simple FBP algorithm is applied to reconstruct CT image. Fig.1. shows the whole flow chart of the algorithm.

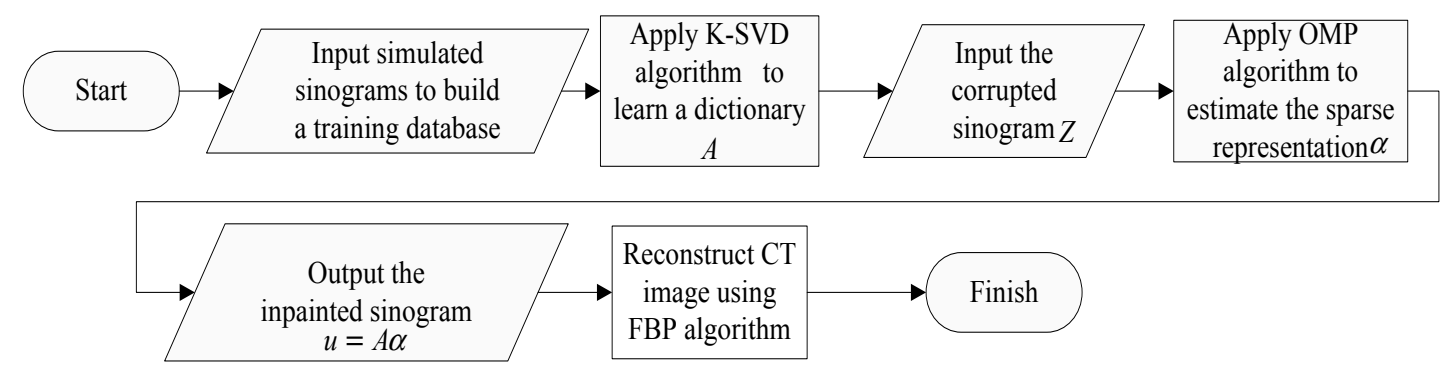

Fig.1. The flow chart of the proposed algorithm 


\subsection{Orthogonal Matching Pursuit}

To obtain the solution of equation (3), we use OMP algorithm to calculate the sparse coefficient $\alpha$ [24]. We consider the unknown $\alpha$ from two aspects- the support of the solution and the non-zero values over this support. We treat $M A$ in (3) as a whole matrix $A^{\prime}$. The OMP algorithm was used to solve the solution of (4):

$$
\min _{\alpha}\|\alpha\|_{0}^{0} \text { s.t. }\|Z-A \alpha\|_{2}^{2} \leq \delta
$$

Fig.2. shows the flow chart of OMP algorithm. In initialization, we initialize $k=0$ ( $k$ is the iteration index), and set the initial solution $\alpha^{0}=0$, the initial residual $r^{0}=Z-A^{\prime} \alpha^{0}=Z$, the initial solution support $S^{0}=$ support $\left\{\alpha^{0}\right\}$.

In the next main iteration stage, we increase $k$ by 1 and perform the following steps:

Sweep: we calculate the error of the following form:

$$
\varepsilon(j)=\min _{M_{j}}\left\|a_{j}^{\prime} M_{j}-r^{k-1}\right\|_{2}^{2}
$$

$M_{j}$ is a scalar, $a_{j}^{\prime}$ is the $j$ th column of $A^{\prime}, r^{k-1}$ is the residual in the $(k-1)$ th iteration:

$$
r^{k-1}=Z-A \alpha^{k-1}
$$

Minimizing of $\varepsilon(j)$ leads to the optimal choice:

$$
M_{j}^{*}=a_{j}^{\prime T} r^{k-1} /\left\|a_{j}^{\prime}\right\|_{2}^{2}
$$

Then $\varepsilon(j)$ can be deduced as following :

$$
\varepsilon(j)=\left\|r^{k-1}\right\|_{2}^{2}-\left(a_{j}{ }^{T} r^{k-1}\right)^{2} /\left\|a_{j}\right\|_{2}^{2}
$$

If one normalized column of the matrix $A$ lead to the largest inner product (in absolute value) between the residual $r^{k-1}$ and the normalized vectors of the matrix $A^{\prime}$, namely the second component in (8) is the largest, then the error $\varepsilon(j)$ will be the minimum.

Update support: after the sweep step, we have calculated the inner product between all the normalized column vectors of $A$ and $r^{k-1}$ to find a minimizer, $j_{0}$ of $\varepsilon(j): \forall j \notin S^{k-1}, \varepsilon\left(j_{0}\right) \leq \varepsilon(j)$ and then we update $S^{k}=S^{k-1} \mathrm{U}\left\{j_{0}\right\}$.

Update provisional solution: compute $\alpha^{k}$ by minimizing of $\left\|A^{\prime} \alpha-Z\right\|_{2}^{2}, \alpha$ is subject to support $\{\alpha\}=S^{k}$.

Update residual: compute $r^{k}=Z-A \alpha^{k}$. If $\left\|r^{k}\right\|_{2}^{2}<\delta$, the algorithm stops, otherwise, we apply another iteration. The output solution $\alpha^{k}$ is obtained after $k$ iterations.

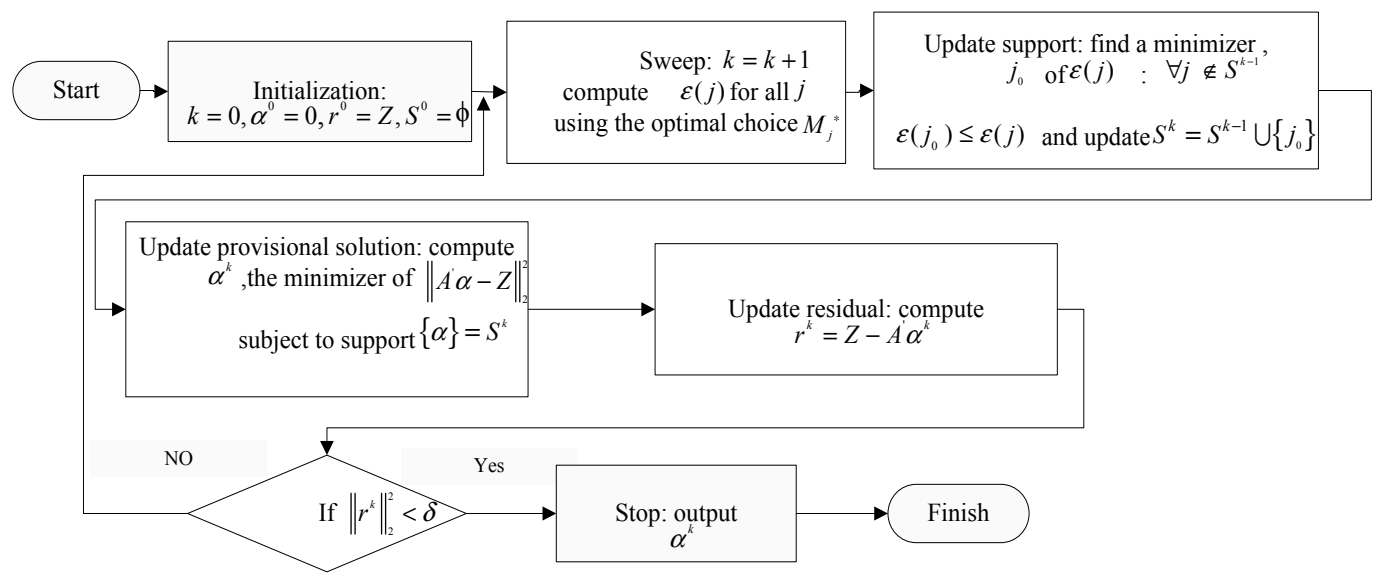

Fig.2. The flow chart of OMP algorithm 


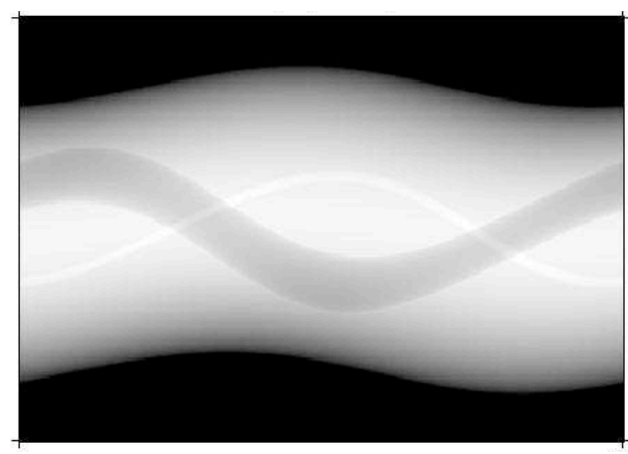

(a)

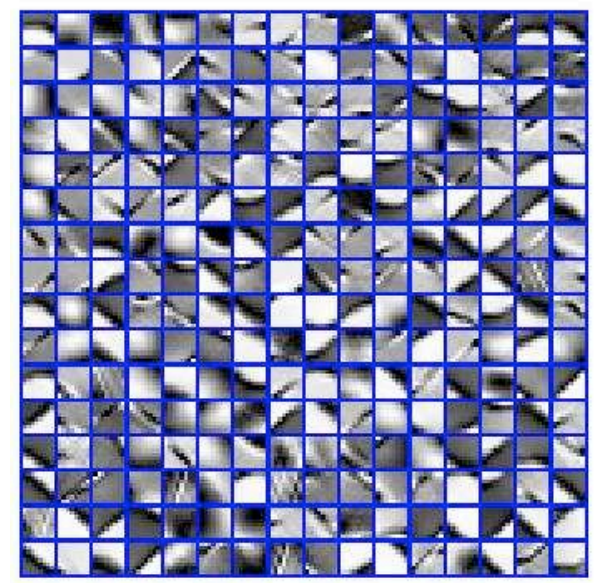

(b)

Fig.3. Simulated sinogram and learnt dictionary. (a): simulated sinogram (b): trained dictionary.

\subsection{Dictionary Learning}

In this study, considering the inherent sinusoidal features for all sinogram data in tomography, we use a simulated sinogram from simulated numeric image to build database $\left\{u_{i}\right\}_{i=1}^{N}$ with $N$ signals, each signal was a patch extracted from the simulated sinogram, then the database was used to learn the dictionary. Fig.3. (a) shows the simulated sinogram from which the learnt dictionary Fig.3. (b) is learned. Every representation $\alpha_{i}$ has $k_{0}$ or fewer non-zero entries. Dictionary $A$ is learned by solving the following optimization problem:

$$
\min _{A,\left\{\alpha_{i}\right\}_{i=1}^{N}} \sum_{i=1}^{N}\left\|u_{i}-A \alpha_{i}\right\|_{2}^{2} \text { s.t. }\left\|\alpha_{i}\right\|_{0} \leq k_{0}, 1 \leq i \leq N
$$

where, each given signal $u_{i}$ is described as the sparsest representation $\alpha_{i}$ over the unknown dictionary $A$. Here the K-SVD algorithm in [15] is applied to learn a dictionary. The K-SVD algorithm performs dictionary update in a sequential manner where each column of $A$ is updated jointly with the corresponding representation coefficients. We perform inpainting on overlapped patches extracted from the corrupted sinogram images. The resulting inpainted sinogram is obtained by averaging the overlapped patches, and then can be used in the final FBP reconstruction.

\section{Experiment}

Reconstructions with both simulated data and real scanned phantom data were performed. In order to make comparison, we also use the traditional spline interpolation in [25] to recover the sinogram. Spline interpolation is a form of interpolation where the interpolation is a special type of piecewise polynomial called a spline [25], here we use the 3rd order B-spline interpolation. As to the dictionary learning in the proposed method, refer to the sparse-based image denoising work [14], [22], the patch size is set to be $8 \times 8$ and the number of the dictionary columns is $256, k_{0}$ and iteration number are set to be 3 and 30 respectively, and the initial dictionary is obtained from the discrete cosine transform (DCT). All the parameters are listed in Table 1. Hanning window was selected for all the FBP reconstructions. A PC with Intel I5 CPU and 4G RAM is used as the workstation for experiments.

Table 1. Parameters used in the methods

\begin{tabular}{|c|c|}
\hline Methods & Parameters \\
\hline Spline interpolation & order $=3$, spline type $:$ B-spline \\
\hline Proposed method & patch size $=8 \times 8$, dictionary size $=64 \times 256, k_{0}=3$, iteration number $=30$ \\
\hline
\end{tabular}




\subsection{Simulated Phantom Data}

In this experiment, we use the $128 \times 128$ Shepp-Logan numeric phantom with pixels intensities ranging from 0 to 255 . The corresponding projected parallel sinogram is with 180 projection views and 128 bins (Fig.4. (a)). Gaussian noise with $\sigma=5$ was added into the sinogram to simulate the practical scanning. We take samples at every 4 degrees in the range of 180 , and the simulated sparse sinogram is illustrated in Fig.4. (b).The recovered sinograms with the two different methods are depicted in Fig.4. (c)-(d) for spline interpolation and the proposed sparse inpainting, respectively. Fig.5. plots the profile and its partial enlargement in the middle line along the horizontal direction of the recovered sinograms. We can see that, compared to spline interpolated sinogram, sparse inpainted sinogram is closer to the original complete one (see the zoomed region in Fig.5.). Fig.6. shows the reconstruction results with zoomed regions, and we can see the sparse inpainted sinogram yields the reconstructed image closer to the original phantom image than spline interplolated sinogram. We can also observe some remaining artifacts in the image reconstructed from the proposed sparse inpainted sinogram, but obviously much less than the reconstruction using spline interpolated sinogram.
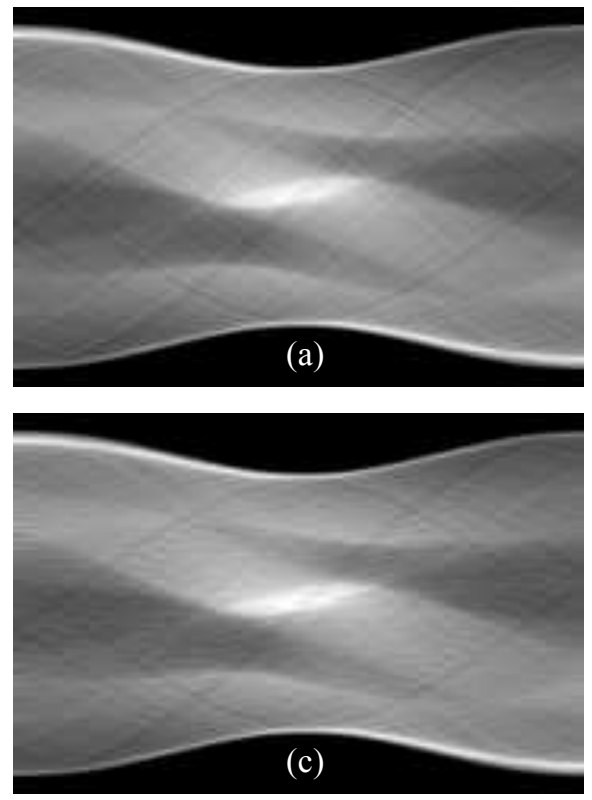
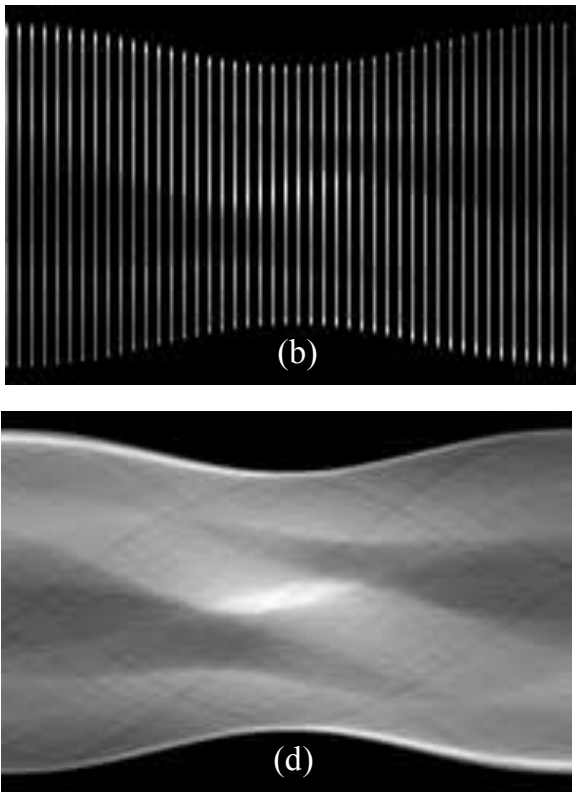

Fig.4. The original sinogram and the recovered sinograms using different methods (a):original sinogram (b):sparse sampled sinogram (c):spline interpolated sinogram (d): sparse inpainted sinogram

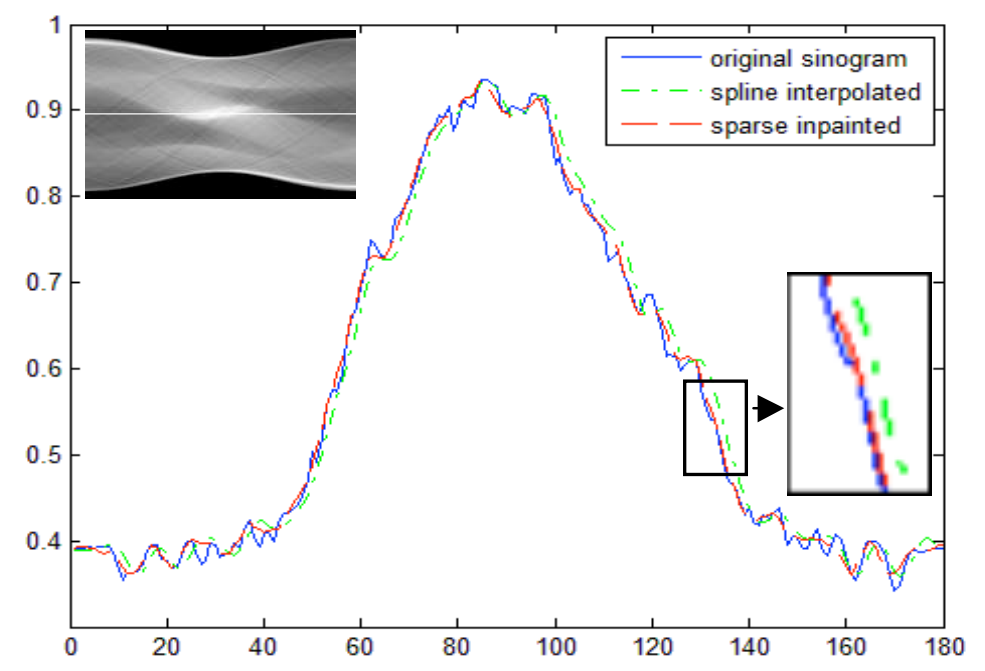

Fig.5. 1D Profiles along the specified horizontal direction (white line) in the recovered sinograms 

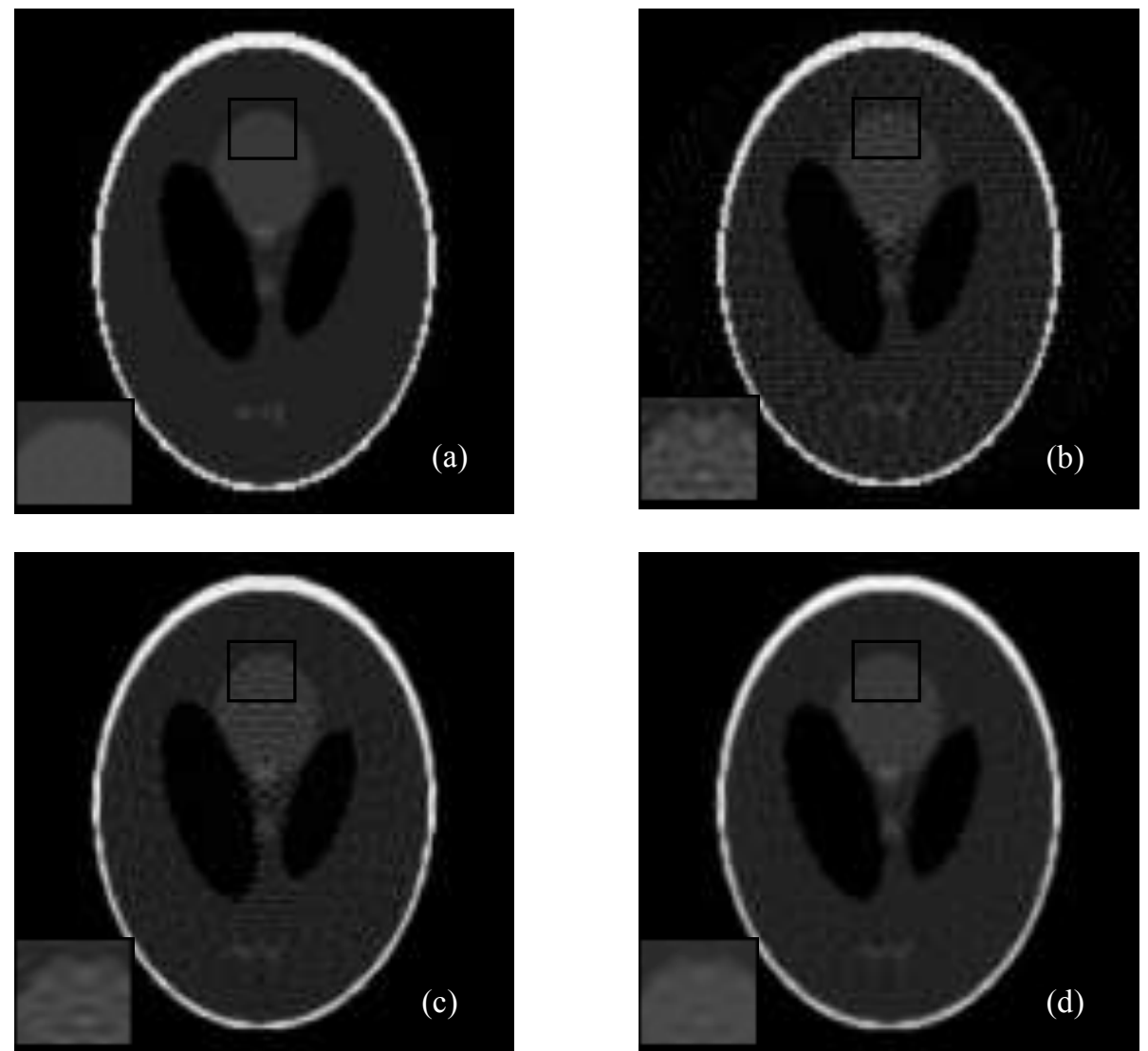

Fig.6. FBP reconstructions using (a): the original sinogram (b): the sparse sampling data (c) the spline interpolated sinogram (d): the sparse inpainted sinogram

\subsection{Real Data}

The real projected sinogram data (Fig.7.(a)) was collected by scanning a phantom from a real fan beam CT system with $120 \mathrm{kVp}$ and $100 \mathrm{~mA}$. The sinogram is with 984 projection views and 888 bins (Fig.7.(a)), and we take one sample per 4 degrees to simulate the sparse scanned sinogram (Fig.7.(b)). The objective image is composed by $512 \times 512$ pixels. Here we consider the abdomen window (center, $50 H U$; window width, 350HU).

Inpainted sinograms with the two different methods are depicted in Fig.7.(c)-(d) for spline interpolation and the proposed sparse inpainting, respectively. Fig.8. plots the profile and its partial enlargement along the chosen horizontal profiles in the two recovered sinograms, and it shows that sparse inpainted sinogram is closer to the original complete one (see the zoomed regions). Fig.9. shows the corresponding reconstruction results with zoomed specified regions. Also in Fig.9., the visual comparisons indicate that for the real data our proposed method can lead to reconstructed image closer to the reconstruction using the original complete sinogram. 

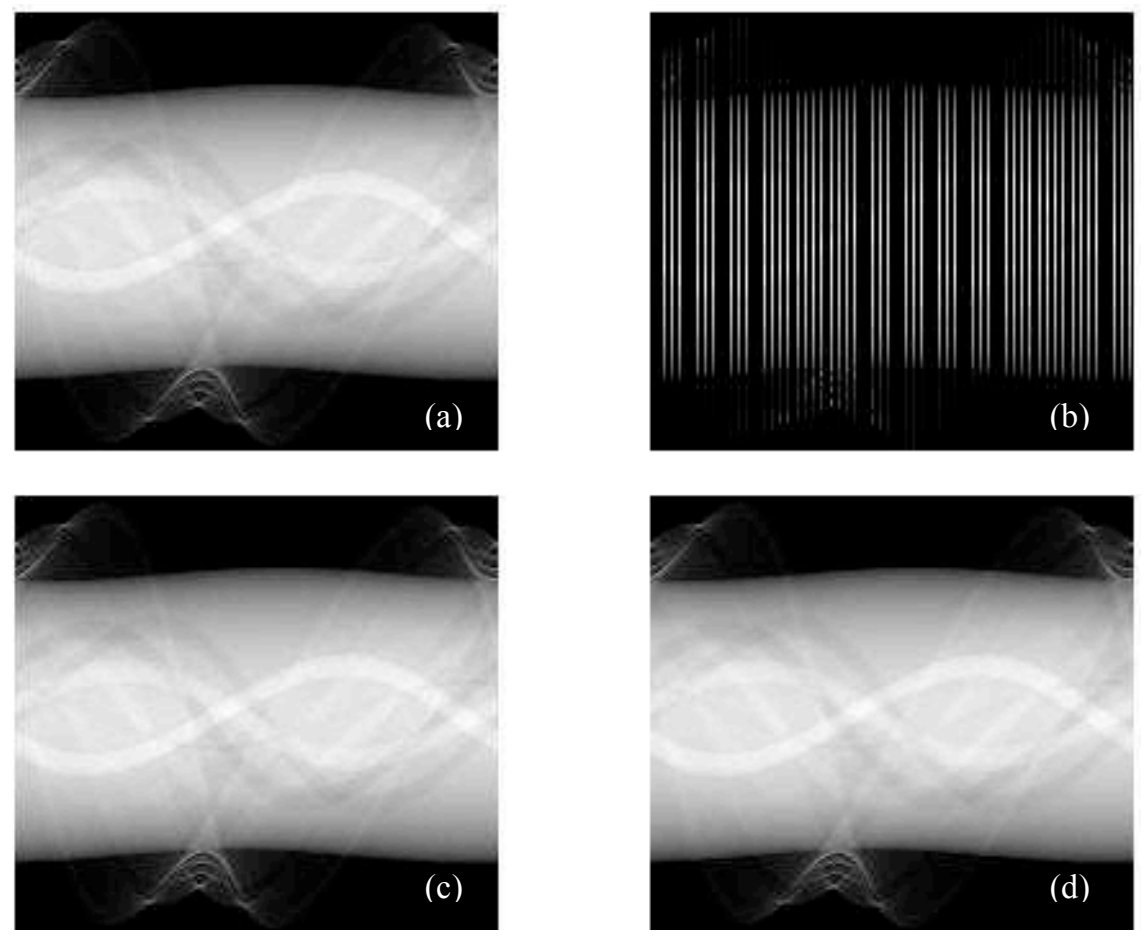

Fig.7. The original sinogram and the recovered sinograms using different methods (a):original sinogram (b):sparse sampled sinogram (c):spline interpolated sinogram (d): sparse inpainted sinogram

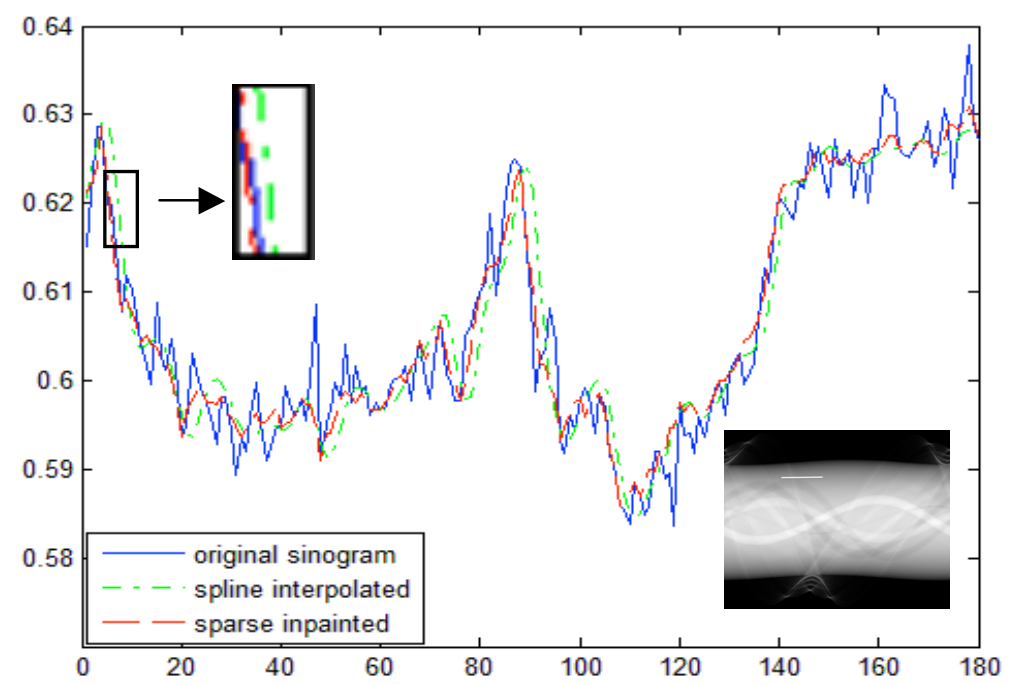

Fig.8. 1D Profiles along the specified horizontal direction (white line) in the recovered sinograms 

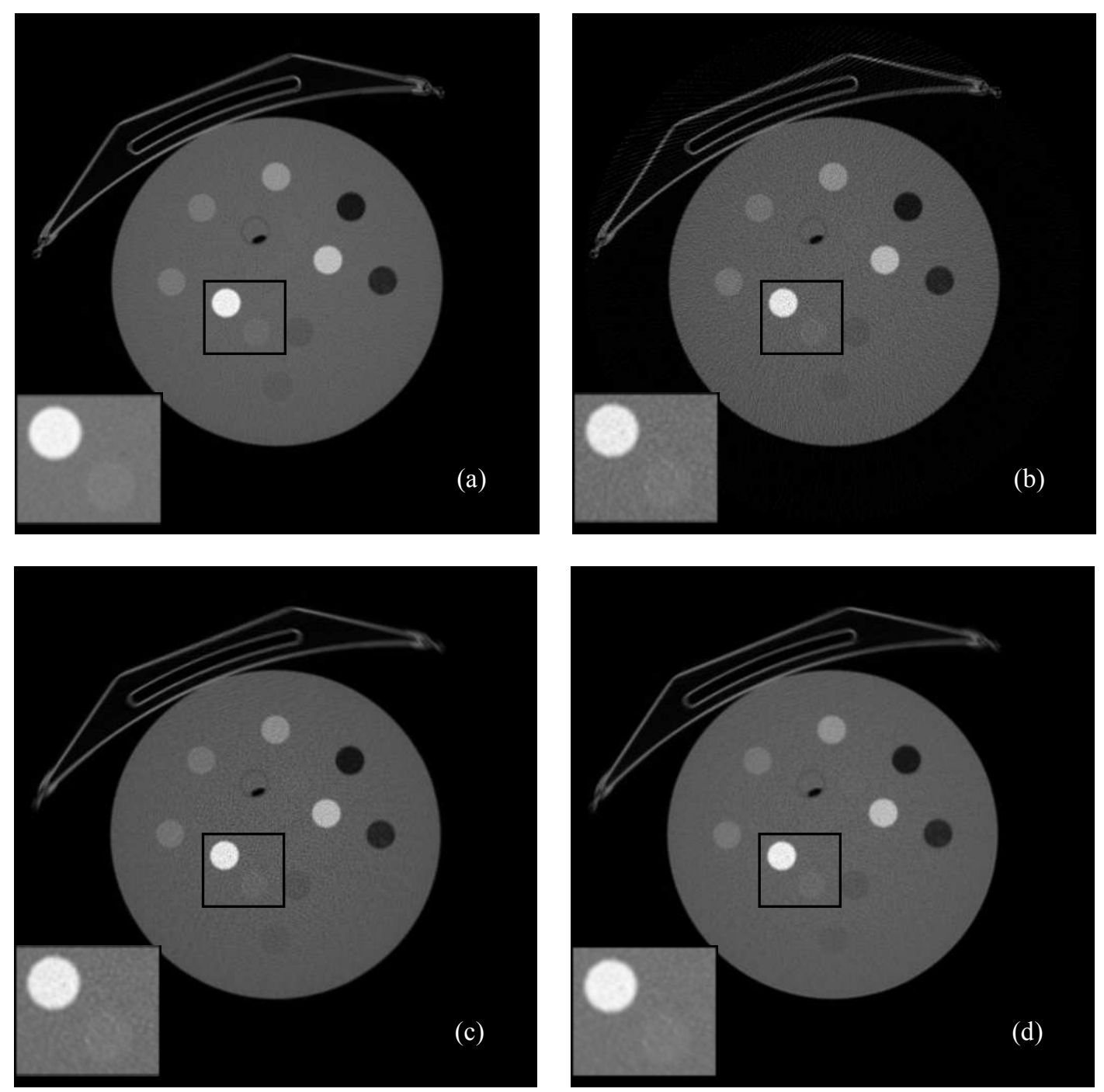

Fig.9. FBP reconstructions using (a): the original sinogram (b): the sparse sampling data (c): the spline interpolated sinogram (d): the sparse inpainted sinogram

\subsection{Quantitative Analysis}

In Table 2, we list the metric peak signal to noise ratio (PSNR) and the structural similarity index comparisons (SSIM) of the recovered sinograms and the reconstructed images for the two experiments on simulated and real data [26]. We take the original complete sinograms and the corresponding reconstructed images as the references. $I$ represents the reference sinogram or image, and $K$ represents the sinogram or image to be assessed.

$$
\begin{gathered}
M S E=\frac{1}{m n} \sum_{i=0}^{m-1} \sum_{j=0}^{n-1}[I(i, j)-K(i, j)]^{2} \\
P S N R=10 \log 10\left(\frac{M A X_{I}^{2}}{M S E}\right)
\end{gathered}
$$

$M A X_{I}$ is the maximum intensity of the reference image $I$.

$$
\operatorname{SSIM}(x, y)=\frac{\left(2 \mu_{x} \mu_{y}\right)\left(2 \sigma_{x y}+c_{2}\right)}{\left(\mu_{x}^{2}+\mu_{y}^{2}+c_{1}\right)\left(\sigma_{x}^{2}+\sigma_{y}^{2}+c_{2}\right)}
$$

where $\mu_{x}$ and $\mu_{y}$ denote the mean intensities of images $x$ and $y, \sigma_{x}$ and $\sigma_{y}$ are the standard deviation of images $x$ and $y, \sigma_{x y}$ is the covariance of $x$ and $y, c_{1}=\left(K_{1} L\right)^{2}$ and $c_{2}=\left(K_{2} L\right)^{2} . L$ is the dynamic 
range of the pixel values (255 for 8-bit grayscale images), $K_{1}=0.01$ and $K_{2}=0.03$. We can see in Table 3. that, for the phantom and real data, our proposed method lead to sinograms and reconstructed images with a higher PSNR and SSIM than spline interpolated method.

Also the CPU time for different reconstructions is displayed in Table 3. We can note that the computation cost for the sparse inpainting method (including dictionary learning and sinogram inpainting) is comparably higher than the spline interpolation. Considering the dictionary can be learned from an available simulated sinogram, the calculation involved in dictionary learning can be avoided in practical application.

Table 2. PSNR(dB)/SSIM values of the two methods

\begin{tabular}{|c|c|c|c|}
\hline \multicolumn{2}{|c|}{} & Spline interpolated & Sparse inpainted \\
\hline \multirow{2}{*}{$\begin{array}{c}\text { Simulated } \\
\text { data }\end{array}$} & Recovered sinograms & $32.8744 / 0.8852$ & $33.9031 / 0.9306$ \\
\cline { 2 - 4 } & Reconstructed images & $28.0298 / 0.8474$ & $30.2174 / 0.9192$ \\
\hline \multirow{2}{*}{ Real data } & Recovered sinograms & $43.7997 / 0.9761$ & $46.9594 / 0.9850$ \\
\cline { 2 - 4 } & Reconstructed images & $34.6735 / 0.9014$ & $38.4771 / 0.9334$ \\
\hline
\end{tabular}

Table 3. CPU time (in seconds) needed for the spline interpolation and the sparse inpainting

\begin{tabular}{|c|c|c|c|c|}
\hline \multicolumn{2}{|c|}{} & Spline interpolation & \multicolumn{2}{|c|}{ Sparse inpainting } \\
\hline \multirow{2}{*}{ CPU time } & \multirow{2}{*}{ Simulated data } & 0.2373 & $\begin{array}{c}\text { Dictionary } \\
\text { learning }\end{array}$ & 262.91 \\
\cline { 2 - 5 } & \multirow{2}{*}{ Real data } & \multirow{2}{*}{25.4530} & $\begin{array}{c}\text { Sinogram } \\
\text { Inpainting }\end{array}$ & 115.64 \\
\cline { 3 - 5 } & & & $\begin{array}{c}\text { Dictionary } \\
\text { learning }\end{array}$ & 333.59 \\
\cline { 3 - 5 } & & & $\begin{array}{c}\text { Sinogram } \\
\text { Inpainting }\end{array}$ & 350.71 \\
\hline
\end{tabular}

\section{Conclusion}

In this paper, a dictionary based sinogram inpainting method is proposed to compensate the artifact problem in CT sparse reconstruction. The patch-based dictionary is first learned by applying K-SVD algorithm with database composed by the patches from simulated CT sinogram. The learned dictionary is then used to inpaint the missing sinogram data which is then used in final image reconstruction via the classical FBP reconstruction. Experiments on both simulated and real data demonstrate the good performance of the proposed inpainting algorithm over other interpolation methods. This algorithm can enable fast scanning with a reduced x-ray dose and also can be easily extended to cone-beam CT system.

However, the proposed inpaining method requires more intensive computation than other approaches, and we can still observe some artifact in the images reconstructed from the proposed sparse inpainted sinogram. Future work includes accelerating the computation using parallelization methods, and improving the inpainting by investigating more elaborate methods on sparse coding.

\section{References}

[1] D.J. Brenner, E.J. Hall, Computed Tomography-An Increasing Source of Radiation Exposure. $N$ Engl J Med, vol.357, 2007, pp. 2277-2284.

[2] Y. Chen, W.F. Chen,et.al., Improving Low-dose Abdominal CT Images by Weighted Intensity Averaging over Large-scale Neighborhoods. European Journal of Radiology, vol.80, no.2, 2011, pp. 42-49.

[3] Y. Chen, Z. Yang, Y.N. Hu, G.Y. Yang, L.M. Luo, W.F. Chen,et.al., Thoracic Low-dose CT Image Processing Using An Artifact Suppressed Large-Scale Nonlocal Means. Physics in Medicine and Biology, vol.57, 2012, pp. 2667-2688. 
[4] Y.S. Li, Y. Chen, L.M. Luo,et.al., A Strategy of CT Sinogram Inpainting Based on Sinusoid-Like Curve Decomposition and Eigenvector-Guided Interpolation. J. Opt. Soc. Am. A, vol.29, no.1, 2012, pp, 153-163.

[5] E.Y. Sidky, C.M. Kao, and X. Pan, Accurate Image Reconstruction from Few-Views and LimitedAngle Data in Divergent-Beam CT. J.X-ray Sci. Tech, vol.14, 2006, pp. 119-139.

[6] Y. Chen, Q. Feng, L.M. Luo, P. Shi, W. Chen, Nonlocal Prior Bayesian Tomographic Reconstruction. J Math Imaging Vision, vol.30, 2008, pp.133-146.

[7] J. Tang, B. Nett and G. Chen, Performance Comparison Between Total Variation(TV) - Based Compressed Sensing and Statistical Iterative Reconstruction Algorithms. Physics in Medicine and Biology, vol.54, 2009, pp. 5781-5804.

[8] J.C. Carr, W.R. Fright, R.K. Beatson, Surface Interpolation with Radial Basis Functions for Medical Imaging. IEEE Transactions on Medical Imaging, vol.16, 1997, pp. 96-107.

[9] H. Kostler, M. Prummer, U. Rude, Adaptive Variational Sinogram Interpolation of Sparsely Sampled CT Data. ICPR 2006.18th International Conference on Pattern Recognition, vol.3, 2006, pp:778-781.

[10] M.S. Lewic, B.A. Olshausen, D.J. Field, Emergence of Simple-cell Receptive Field Properties by Learning a Sparse Code for Natural Images. Nature, vol. 381, 1996, pp. 607-609.

[11] M.S. Lewicki, Learning Overcomplete Representations. Neural Comput, vol.12, 2000, pp.337-365.

[12] K.K. Delgado, J.F. Murray, et.al., Dictionary Learning Algorithms for Sparse Representation. Neural Comput, vol.15, 2003, pp.349-396.

[13] D.L. Donoho and M. Elad, Maximal Sparsity Representation via L1 Minimization. Proc. Nat. Aca. Sci, vol.100, 2003, pp. 2197-2202.

[14] M. Elad and M. Aharon, Image Denoising via Sparse and Redundant Representations over Learned Dictionaries. IEEE Transactions on Image Processing, vol.15, 2006, pp. 3736-3745.

[15] M. Aharon, M. Elad, A.M. Bruckstein, The K-SVD: An Algorithm for Designing of Overcomplete Dictionaries for Sparse Representation. IEEE Trans. on Signal Processing, vol.54, no.11, November 2006, pp.4311-4322.

[16] J. Mairal, G. Sapiro, and M. Elad, Learning Multiscale Sparse Representations for Image and Video Restoration. SIAM Multiscale Modeling and Simulation, vol.7, 2008, pp. 214-241.

[17] M.J. Fadili, J.L. Starck, F. Murtagh, Inpainting and Zooming Using Sparse Representations. The Computer Journal, vol.52, no.1, 2009, pp.64-79.

[18] J. Wright, A.Y. Yang, A. Ganesh, S.S. Sastry, and Y. Ma, Robust Face Recognition via Sparse Representation. IEEE Transactions on Pattern Analysis and Machine Intelligence, vol.31, 2008, pp. 210-227.

[19] S. Ravishankar and Y. Bresler, MR Image Reconstruction from Highly Undersampled K-space Data by Dictionary Learning. IEEE Trans. Med. Imag, vol.30, 2012, pp. 1028-1041.

[20] Q. Xu, H.Y. Yu, X.Q. Mou, L. Zhang, J. Hsieh, G. Wang, Low-Dose X-ray CT Reconstruction via Dictionary Learning. IEEE Trans. Med. Imag, vol.31, 2012, pp.1682-1697.

[21] S. Li, L. Fang, and H. Yin, An Efficient Dictionary Learning Algorithm and Its Application to 3-D Medical Image Denoising. IEEE Trans. Biomed. Eng, vol.59, 2012, pp. 417-427.

[22] J. Shtok, M. Elad, M. Zibulevsky, Sparsity-Based Sinogram Denosing for Low-Dose Computed Tomography. International Conference on Acoustics, Speech and Signal Processing, Prague, Czech Republic,22-27 May,2011.

[23] A.C. Kak, M. Slaney, Principles of Computerized Tomographic Imaging. New York :IEEE Press, 1998.

[24] V.N. Temlyakov, Greedy Algorithms and M-term Approximation. Journal of Approximation Theory, vol.98, 1999, pp.117-145.

[25] E.B. Dam, M. Koch, M. Lillholm, Quaternion Interpolation and Animation. Technical Report DIKUTR-98/5,1998.

[26] Z. Wang, A.C. Bovik, H.R. Sheikh, Image Quality Assessment: From Error Visibility to Structural Similarity. IEEE Transactions on Image Processing, vol.13, no.4, 2004, pp.600-612. 